\title{
Evaluación de la incapacidad provocada por el tinnitus: homologación lingüística nacional del Tinnitus Handicap Inventory (THI)
}

\author{
Evaluation of discapacity generated by tinnitus: national linguistic \\ homologation of Tinnitus Handicap Inventory (THI) \\ Handicap Evaluation in Tinnitus Patients: a national linguistic adaptation
}

\author{
Alejandro Peña Martínez ${ }^{1}$
}

\begin{abstract}
RESUMEN
La evaluación del tinnitus neurosensorial es de suma importancia para el estudio de los pacientes que la padecen; existen dos maneras de efectuar dicho estudio, primero la acufenometría que comprende la confrontación de frecuencia y de intensidad, la enmascarabilidad del tinnitus y la búsqueda de la inhibición residual y segundo, la más importante, la medición psico-emocional. 1 test más conocido y valorado para este propósito es el Tinnitus Handicap Inventory (THI). En el presente artículo se propone una homologación lingüística nacional de dicho test que permita obtener respuestas confiables y veraces.

Palabras claves: Test de incapacidad del tinnitus, $7 H$, Questionario del Tinnitus.
\end{abstract}

\section{SUMMARY}

The evaluation of neurosensorial tinnitus is highly important in the study of these patients; thereare two ways of conducting this study, firstly tinnitometry, which involves the confrontation of frequency and intensity, the maskability of tinnitus and the search for the residual inhibition and secondly and most important, psycho-emotional medicine. The best known and valued test for this purpose is the Tinnitus Handicap Inventory (THI). In this article, a national linguistic homologation of this test is proposed, that allows for reliable and truthful answers.

Key words: Tinnitus Handicap Inventory, THI, Tinnitus Questionnaire.

\section{INTRODUCCIÓN}

La evaluación del tinnitus neurosensorial o acúfenos es de primordial importancia para el médico o investigador que requiera medir el impacto de este síntoma, en un paciente o grupo de pacientes, en los que desee conocer el efecto de tal o cual terapia, o confrontar o comparar resultados terapéuticos y para efectuar el seguimiento de aquellos pacientes afectados por tal síntoma. Para

\footnotetext{
${ }^{1}$ Médico Aorrinolaringólogo. Talca
} 
cumplir con tales objetivos debe necesariamente seguirse dos caminos, primero se requiere efectuar los llamados test psicoacústicos: acufenometría o tinnitumetría, y segundo, y lo más importante, evaluar la repercusión psicológica que dicho síntoma le provoca al paciente, a través de los llamados test psicoemocionales; al respecto a Fowler $^{1}$ el año 1942, le llamaba la atención al efectuar las primeras tinnitumetrías la pequeñez de la intensidad del acúfeno medido en $\mathrm{dB}$, al compararlo con la gran repercusión mental que este síntoma le provocaba a algunos de los pacientes.

\section{MEDICIÓN PSICOACÚSTICA O ACUFENOMETRÍA}

La medición psicoacústica, acufenometría o tinnitumetría fue estandarizada en 1981 por un panel de expertos reunidos en Londres en un simposio de la Fundación Giba², allí se acordó el cumplimiento de cuatro medidas fundamentales en la descripción del acúfeno: a) confrontación de la frecuencia, b) confrontación de la intensidad, ambas descritas por Fowler $^{3}$, c) la enmascaribilidad del tinnitus, descrita por Feldman ${ }^{4}$ y d) la inhibición residual, descrita por Vernon ${ }^{5}$. Sin embargo la trascendencia de la medición psicoacústica, hoy en día se ha redimensionado, considerando más bien, de primera importancia la severidad con que el paciente experimenta el síntoma y su repercusión mental que puede llevarlo a la depresión o incluso al suicidio 6 .

\section{MEDICIÓN PSICOEMOCIONAL}

Es así como, el otro camino que necesariamente debe seguirse en la evaluación del tinnitus es medir el impacto psicológico y la incapacidad que el acúfenos le provoca al paciente; al respecto el test más aceptado en la literatura anglosajona es el propuesto por Newman y Jacobson en $1996^{7}$ conocido como el Tinnitus Handicap Inventory o por sus siglas $T H I$, el cual hasido aceptado por los principales centros dedicados al estudio del tinnitus por su confiabilidad, seguridad y validez avalado por estudios estadísticos psicométricos ${ }^{8-10}$.
Este test de incapacidad del tinnitus, consiste en 25 preguntas de fácil comprensión para el paciente quien tiene tres opciones de respuesta para cada pregunta: sí, a veces, o no, otorgándole 4 puntos al sí, 2 puntos al a veces, y 0 punto al no, de manera que la suma del puntaje total puede ir de 0 a 100.

日 THl está a su vez subdivido en tres subescalas, la primera, Ilamada Funcional por Newman, compuesta de 11 items, abarcando el área de la función mental por ej.¿debido al tinnitus le es difícil concentrarse?, el área de la función social/ocupacional por ej.¿debido al tinnitus le es difícil disfrutar de actividades sociales como salir a comer o ir al cine?, y el área de la función física por ej. ¿debido al tinnitus le cuesta dormirse en la noche? La segunda subescala es la llamada Emocional compuesta por 9 items que abarcan un amplio rango de respuestas afectivas provocadas por el acúfenos, a saber, rabia, frustración, irritabilidad y depresión. La tercera escala es la llamada Catastrófica, compuesta por 5 items y que reflejan la desesperación del paciente, su incapacidad de poder escapar del problema, su percepción de tener una enfermedad gravísima, la pérdida del control y su incapacidad de enfrentar el problema.

\section{CLASIFICACIÓN DE CALIDAD DE VIDA SEGÚN EL THI}

日 año 1999 la Asociación Británica de Otorrinolaringología comisionó a un grupo de trabajo con el propósito de tener una guía estandarizada de calificación de la severidad del tinnitus. Este grupo encabezado por Mc Combe ${ }^{11}$ clasificó en 5 niveles la incapacidad provocada por el acúfenos: muy leve, leve, moderado, severo y catastrófico.

Grado 1, o muy leve (THI 0 a 16): tinnitus solo percibido en ambiente silencioso y fácilmente enmascarable, y que casi nunca perturba al paciente.

Grado 2, leve (THI 18 a 36): tinnitus enmascarable por el ruido ambiente y olvidado durante la actividad diaria.

Grado 3, moderado (TH 38 a 56): tinnitus percibido a pesar del ruido ambiente, si bien no 
dificulta las actividades diarias; sin embargo molesta en el reposo o la quietud y a veces dificulta la conciliación del sueño.

Grado 4, severo (THI 58 a 76): tinnitus siempre percibido, interfiriendo las actividades diarias, dificultando siempre el reposo y el sueño; estos pacientes acuden frecuentemente a especialistas buscando ayuda.

Grado 5, catastrófico (THI 78 a 100): todos los síntomas son peores que el grado 4 , especialmente el insomnio; es posible encontrar patologías psiquiátricas asociadas

\section{TEST DE INCAPACIDAD DEL TINNITUS}

日 THI ha sido traducido a varios idiomas ${ }^{10,12,13}$ y adaptado según el lenguaje y la idiosincrasia de cada país.

También existe una versión española de Herráiz y col $^{14}$, traducida del inglés y adaptada al lenguaje cotidiano de España, pero que difiere de nuestro lenguaje en sus giros idiomáticos y vocabulario. Por tal motivo parece necesario una homologación lingüística nacional del $T H I$ que sea fácilmente comprensible a todos los estratos socioculturales del país y que nos permita obtener respuestas confiables, veraces y fidedignas de nuestros pacientes (Tabla 1).

Tabla 1. Test de incapacidad del tinitus

\begin{tabular}{|c|c|c|c|c|}
\hline $1 \mathrm{~F}$ & ¿Le cuesta concentrarse por culpa del ruido o zumbido de oído? & Sí & A veces & No \\
\hline $2 F$. & ¿Le cuesta escuchar a los demás debido a que el zumbido es muy fuerte? & Sí & A veces & No \\
\hline $3 F$ & ¿Lo pone mal genio el zumbido del oído? & Sí & A veces & No \\
\hline 4F. & ¿Se siente confundido por culpa del zumbido del oído? & Sí & A veces & No \\
\hline $5 \mathrm{C}$ & ¿Se desespera con el ruido o zumbido del oído? & Sí & A veces & No \\
\hline $6 \mathrm{E}$ & ¿Se queja mucho por tener el zumbido en el oído? & Sí & A veces & No \\
\hline 7F. & ¿Le cuesta quedarse dormido en la noche por culpa del zumbido del oído? & Sí & A veces & No \\
\hline $8 \mathrm{C}$. & ¿Cree que el problema de su zumbido es algo sin solución? & Sí & A veces & No \\
\hline 9F. & ¿曰 zumbido del oído es un problema que le impide disfrutar de la vida como & & & \\
\hline & por ejemplo salir a comer con amigos o ir al cine? & Sí & A veces & No \\
\hline 10E & ¿Se siente desilusionado por culpa del zumbido del oído? & Sí & A veces & No \\
\hline $11 C$. & ¿Oree que tiene un enfermedad incurable? & Sí & A veces & No \\
\hline 12F. & ¿日 zumbido de oído le impide pasarlo bien? & Sí & A veces & No \\
\hline 13F. & ¿Le estorba el zumbido de oído en su trabajo o en las labores de la casa? & Sí & A veces & No \\
\hline 14F. & ¿Se siente a menudo de mal genio por culpa del zumbido del oído? & Sí & A veces & No \\
\hline 15F & ¿Le cuenta comprender lo que lee por culpa del zumbido del oído? & Sí & A veces & No \\
\hline $16 \mathrm{E}$ & ¿Se siente alterado por el zumbido de oído? & Sí & A veces & No \\
\hline $17 \mathrm{E}$ & $\begin{array}{l}\text { ¿Siente que el zumbido de oído ha echado a perder las relaciones con sus } \\
\text { familiares y amigos? }\end{array}$ & Sí & A veces & No \\
\hline 18F. & ¿Le cuesta sacarse de la cabeza el zumbido y concentrarse en otra cosa? & Sí & A veces & No \\
\hline 19C. & ¿Siente que no puede controlar el zumbido de oído? & Sí & A veces & No \\
\hline 20F. & ¿Se siente a menudo cansado por culpa del zumbido de oído? & Sí & A veces & No \\
\hline $21 \mathrm{E}$ & ¿Se siente deprimido por causa del zumbido de oído? & Sí & A veces & No \\
\hline 22E & ¿Lo pone nervioso el zumbido de oído? & Sí & A veces & No \\
\hline 23C. & ¿Siente que no puede ya hacerle frente al zumbido de oído? & Sí & A veces & No \\
\hline 24F. & ¿Empeora el zumbido de oído cuando está estresado? & Sí & A veces & No \\
\hline $25 \mathrm{E}$ & ¿Se siente inseguro por culpa el zumbido de oído? & Sí & A veces & No \\
\hline
\end{tabular}




\section{BIBLIOGRAFÍA}

1. Fomber $\boxminus$. The illusion of loudness of tinnitus its etiology and treatment. Laryngoscope 1942; 52: 275-85.

2. GBA FOUNDATION. Tinnitus. GBA Foundation Symposium 85. London, Pitman Medical, 1981.

3. Fom $\in$ $\boxminus$. Head noises in normal and disordered ear. Arch Otolaryngol 1944; 39: 498-503.

4. FEDMAN H. Homolateral and contralateral masking of tinnitus by noise bands and by pure tones. Audiology 1971 ; 10: 138-144.

5. V'RNON J. Relief of tinnitus by masking Treatment.En: English G eds, Oolaryngology. Philadelphia: J.B. Lippincott Co, 1988; chapter 53.

6. CIDA B, BRIZI P, LUPPI B. Misurazioni psicoacustiche ( acufenometria). En: Ouda D, ed, Acufeni: Diagnosi e Terapia Piacensa: TorGraf, 2004; 97-105.

7. NEMMAN GH, JACOBSON GP, SPIT\&R JB. Development of the Tinnitus Handicap Inventory. Arch Oolaryngol Head Neck Surg 1996; 122: 143-8.

8. BAGULEY DM, ANDERSON G Factor analysis of the Tinnitus Handicap Inventory. Am J Audiol 2003; 12(1): 31-4.
9. NeMman CW, Sandridge SA, JaCobson GP. Psychometric adecuacy of the Tinnitus Handicap Inventory (THI) for evaluating treatment outcome. J Am Acad Audiol 1998; 9 (2): 153-60.

10. ODDA D. Acufeni soggettivi: Aspetti Cinici. En: Cuda D,ed, Acufeni: Diagnosi e Terapia. Piacenza: TorGraf, 2004; 51-60.

11. MoCOMbe A, Baguley D, COLes R et Al. Guidelines for the grading of tinnitus severty: the results of a working group commisioned by the British Association of Otolaryngologists. Allied Sci 2001; 26 (5): 388-93.

12. Paula E CunHa F, ONish Et et al. Tinnitus Handicap Inventory: cross-cultural adaptation to BrazilianPortuguese. ProFono 2005; 17 (3): 303-10.

13. ZAGHARIAS R, MIRZF, JOHANSEn LV et AL. Reliability and validity of a Danish adaptation of the Tinnitus Handicap Inventory. Scand Audiol 2000; 29 (1): 37-43.

14. Herrálz C, Hernández J, Plaza Get al. Evaluación de la Incapacidad en pacientes con acúfenos. Acta Otorrinolaring Esp 2001; 52: 534-8.

Dirección: Dr. Alejandro Peña Martínez

5 Oriente 2249, Talca.

E-mail: pena.martinez@gmail.com 